\title{
Diagnostic accuracy of the standard and alternative periodic leg movement during sleep indices for restless legs syndrome
}

Raffaele Ferri ${ }^{\mathrm{a}, *}$, Francesco Rundo ${ }^{\mathrm{a}}$, Marco Zucconi ${ }^{\mathrm{b}}$, Mauro Manconi ${ }^{\mathrm{c}}$, Debora Aricò ${ }^{\mathrm{a}}$, Oliviero Bruni $^{d}$, Luigi Ferini-Strambi ${ }^{b}$, Stephany Fulda ${ }^{c}$

${ }^{a}$ Sleep Research Centre, Department of Neurology I.C., Oasi Institute for Research on Mental Retardation and Brain Aging (IRCCS), Troina, Italy

${ }^{\mathrm{b}}$ Sleep Disorders Center, Department of Neurology, Scientific Institute and University Ospedale San Raffaele, Vita-Salute University, Milan, Italy

${ }^{c}$ Sleep and Epilepsy Center, Neurocenter of Southern Switzerland, Civic Hospital (EOC) of Lugano, Lugano, Switzerland

${ }^{\mathrm{d}}$ Department of Social and Developmental Psychology, Sapienza University, Rome, Italy

*Corresponding author: Sleep Research Centre, Department of Neurology IC, Oasi Institute (IRCCS), Troina, Italy. Tel.: +39-0935-936111; fax +39-0935-936694.

E-mail address: rferri@,oasi.en.it (Dr Raffaele Ferri) 


\section{Abstract}

Objective: To evaluate the diagnostic accuracy of the standard periodic leg movement during sleep (PLMS) index, a recently introduced alternative one, and the periodicity index (PI) for restless legs syndrome (RLS).

Subjects and methods: A total of 107 patients with RLS were retrospectively identified and included (47 males and 60 females, mean age 56.9 years), along with 48 normal controls (24 males and 24 females, mean age 52.0 years). The standard PLMS index, the alternative PLMS index and the PI were calculated from polysomnographic recordings. In order to establish the best performing threshold for the diagnosis of RLS, sensitivity and specificity were calculated with a series of threshold values ranging from 5-20/hour for the two PLMS indices, and from 0.2-0.9 for the PI.

Results: The optimal threshold was found to be between 15-16/hour for the standard PLMS index, close to 13 /hour for the alternative index, and around 0.5 for the periodicity index. With these thresholds, all methods showed high values of sensitivity and specificity, with corresponding high positive and negative predictive values and accuracy; the ROC areas were similar, and the Kappa coefficient of agreement indicated a 'very good' agreement between the PLMS indices.

Conclusions: This study assessed the cut-off values of the standard (15-16/hour) and alternative (13/hour) PLMS indices, and of the PI (0.5). The diagnostic accuracy of all indices for RLS vs controls was found to be acceptably high, with the new alternative PLMS index performing slightly higher than the other two indices.

\section{Keywords:}

Periodic leg movements during sleep

PLMS index

Restless legs syndrome

Sleep scoring

Diagnostic accuracy 


\section{Introduction}

An alternative periodic leg movement (PLM) index, which more closely reflects the 'genuine' periodic portion of leg movements, has recently been introduced and statistically evaluated [1]. The alternative PLM index adopts the same criteria as the 'standard' PLM index, but only considers leg movements separated by $\geq 10$ seconds and $\leq 90$ seconds and, differently from the standard rules $[2,3]$, interrupts a possible PLM series if the interval between two consecutive LMs is $<10$ seconds [1]. This modification is based on the concern that the standard PLM detection criteria acted as a 'pattern extractor' by ignoring all 'non-fitting' leg movement activity, with the consequence that the PLM index could only increase as the number of LMs increases, which might be problematic in situations where there is a high number of LMs, as the PLM index will no longer reflect the truly periodic LMs but rather the number of LMs [1].

In a previous study [1] of a large group of RLS patients and healthy controls, it was shown that the alternative PLM index was similar to the standard index when leg movement activity was genuinely periodic, as indicated by the Periodicity index [4], but was significantly lower when the periodicity was low. The present study investigated the cut-off value of the alternative index, and its diagnostic accuracy for RLS vs healthy controls, compared with that of the standard index.

\section{Material and methods}

A detailed description of subjects and methods can be found in a previous publication [1]. In short: 107 unmedicated patients with RLS (60 female, 47 male, mean age 56.9 years, SD 12.95) and 48 healthy controls (24 females, 24 males, 52.0 years, SD 19.30), who were matched for age ( $t=1.75$, N.S.) and sex (Chi-square $=0.49$, N.S.), were included. All the participants had an AHI $\leq 5$. All-night polysomnography was carried out according to standard procedures [5]. Leg movements during sleep were identified by automatic detection (Hypnolab) and fully supervised by a human scorer. The standard PLMS index was calculated according to the criteria set by the International RLS Study Group and endorsed by the World Association of Sleep Medicine [2]. The alternative periodic leg movement during sleep (PLMS) index only considered LMs separated by 10-90 seconds. In addition, if the interval between two consecutive LMs was $<10$ seconds, the periodic 


\section{Brief Communication}

sequence was interrupted. In addition, the periodicity index (PI) during sleep $[4,6]$ was computed. For all indices, sensitivity and specificity were calculated for a series of pre-set threshold values, which ranged from 5-20/hour for the standard and the alternative indices, and from 0.2-0.9 for the periodicity index.

\section{Results}

The results are given in Fig. 1, with sensitivity decreasing and specificity increasing with increasing thresholds. The intersection between these two represents the best choice for a threshold to be used for diagnostic purposes. This optimal threshold was between 15-16/hour for the standard PLMS index, and close to 13/hour for the alternative index (Fig. 1). For the periodicity index, the optimal threshold was around 0.5 . Table 1 reports the common parameters of diagnostic accuracy for the standard and the alternative PLMS index, and the periodicity index, with thresholds of $15 /$ hour, 13/hour, and 0.5, respectively. All methods showed high values of sensitivity and specificity, with corresponding high positive and negative predictive values and accuracy. The alternative PLMS index tended to show marginally higher values. In addition, the ROC areas were similar and the Kappa coefficient of agreement indicated a 'very good' agreement between the standard and alternative PLMS index, while there was a lower, but still 'good', agreement of the periodicity index with these measures (Table 1).

\section{Discussion}

A validation process of the alternative PLMS/PLMW index [1], described above, has recently been introduced and successfully initiated. It has been proven to be more adherent to the parameters that allow a reliable evaluation of LM periodicity. Although encouraging, additional validation and analysis of this algorithm's performance are needed and, along with the present study, by evaluating its accuracy for the diagnosis of RLS a further important piece of information will have been added. The present results show this being high, at least at the same level of that of the standard index.

It is important to mention that this analyses has also been undertaken for the standard method, and this data-driven approach has demonstrated that the arbitrary threshold of 15 PLMS/hour of sleep, as used in the AASM International Classification of Sleep Disorders [7], is in good approximation to the empirically found threshold in the present study. It is believed that there are no 


\section{Brief Communication}

other data in previous literature supporting the indication of this threshold. However, as far as the diagnosis of RLS is concerned, the thresholds of 15/hour for the standard PLMS index and of 13/hour for the new alternative index seem to represent a reliable and data-driven choice.

Importantly, this study cannot take any position with regard to the level of PLMS that might have a 'clinical significance', which has often been indicated with a value of $5 /$ hour or has been used in genetic studies of PLMS [8]. It is also noteworthy to state that the performance of all three indices was largely equivalent.

In conclusion, the cut-off value of the alternative PLMS index and its diagnostic accuracy for RLS vs controls was assessed; this was found to be acceptably high (at least comparable to that of the standard index and the periodicity index, if not higher). This strengthens the proposition to use the new PLMS index as a valid alternative to the standard algorithm. Future studies should test the alternative PLMS index in a different dataset than the one used to derive it, evaluate it in younger subjects (children), and explore its application in other clinical samples of patients with frequent PLMS, such as REM sleep behavior disorder and narcolepsy $[9,10]$.

\section{Acknowledgments}

Disclosure statement: This was not an industry-supported study. This work was performed at the Sleep Research Centre, Department of Neurology IC, Oasi Institute (IRCCS), Troina, Italy. This study (Drs Ferri, Aricò, and Rundo) was partially supported by a grant of the Italian Ministry of Health ('Ricerca Corrente'). Drs Manconi and Fulda are supported by the Swiss National Science Foundations (Grant No.:320030_144007). The authors have indicated no financial conflicts of interest. 


\section{References}

[1] Ferri R, Rundo F, Zucconi M, Manconi M, Aricò D, Bruni O, et al. Putting the periodicity back into the PLM Index: an alternative data-driven algorithm for the computation of the Periodic Leg Movement Index during sleep and wakefulness. Sleep Med 2015;16:1229-35.

[2] Zucconi M, Ferri R, Allen R, Baier PC, Bruni O, Chokroverty S, et al. The official World Association of Sleep Medicine (WASM) standards for recording and scoring periodic leg movements in sleep (PLMS) and wakefulness (PLMW) developed in collaboration with a task force from the International Restless Legs Syndrome Study Group (IRLSSG). Sleep Med 2006;7:175-83.

[3] Berry RB, Brooks R, Gamaldo CE, Harding SM, Marcus CL, Vaughn BV. The AASM Manual for the Scoring of Sleep and Associated Events: Rules, Terminology and Technical Specifications, Ver. 2.0. Darien, IL: American Academy of Sleep Medicine; 2012.

[4] Ferri R, Zucconi M, Manconi M, Plazzi G, Bruni O, Ferini-Strambi L. New approaches to the study of periodic leg movements during sleep in restless legs syndrome. Sleep 2006;29:75969.

[5] Iber C, Ancoli-Israel S, Chesson AL, Quan SF. The AASM manual for the scoring of sleep and associated events: rules, terminology, and technical specifications. 1st ed. Westchester, IL: American Academy of Sleep Medicine; 2007.

[6] Ferri R. The time structure of leg movement activity during sleep: The theory behind the practice. Sleep Med 2012;13:433-41.

[7] American Academy of Sleep Medicine. International classification of sleep disorders. 3rd ed. Darien, IL: American Academy of Sleep Medicine; 2014.

[8] Stefansson H, Rye DB, Hicks A, Petursson H, Ingason A, Thorgeirsson TE, et al. A genetic risk factor for periodic limb movements in sleep. N Engl J Med 2007;357:639-47.

[9] Ferri R, Zucconi M, Manconi M, Bruni O, Ferini-Strambi L, Vandi S, et al. Different periodicity and time structure of leg movements during sleep in narcolepsy/cataplexy and restless legs syndrome. Sleep 2006;29:1587-94.

[10] Manconi M, Ferri R, Zucconi M, Fantini ML, Plazzi G, Ferini-Strambi L. Time structure analysis of leg movements during sleep in REM sleep behavior disorder. Sleep 2007;30:177985. 
Table 1. Analysis of the accuracy of the standard and alternative periodic leg movement during sleep (PLMS) indices and of the periodicity index for the diagnosis of restless legs syndrome vs controls.

\begin{tabular}{|c|c|c|c|}
\hline & $\begin{array}{c}\text { Standard PLMS } \\
\text { index (threshold } \\
15 / \text { hour) }\end{array}$ & $\begin{array}{l}\text { Alternative PLMS } \\
\text { index (threshold } \\
13 / \text { hour) } \\
\end{array}$ & $\begin{array}{l}\text { Periodicity index } \\
\text { (threshold 0.5) }\end{array}$ \\
\hline Sensitivity, \% & 83.3 & 86.9 & 84.1 \\
\hline Specificity, \% & 86.0 & 85.4 & 81.3 \\
\hline $\begin{array}{l}\text { Positive predictive value, } \\
\%\end{array}$ & 92.0 & 93.0 & 90.9 \\
\hline $\begin{array}{l}\text { Negative predictive value, } \\
\%\end{array}$ & 72.7 & 74.5 & 69.6 \\
\hline Accuracy, \% & 85.2 & 86.5 & 83.2 \\
\hline ROC area & 0.847 & 0.862 & 0.827 \\
\hline \multicolumn{4}{|c|}{$\begin{array}{l}\text { Alternative vs standard PLMS Index: Kappa }=0.944(95 \% \text { confidence interval } 0.889-0.998) \text {; } \\
\text { Alternative PLMS index vs periodicity index: Kappa }=0.783(95 \% \text { confidence interval } 0.683- \\
0.883) \text {; Standard PLMS index vs periodicity index: Kappa }=0.761(95 \% \text { confidence interval } 0.655- \\
0.868)\end{array}$} \\
\hline
\end{tabular}


Fig. 1. Scoring criteria for the standard and alternative periodic leg movement during sleep indices (PLMS) and periodicity index.
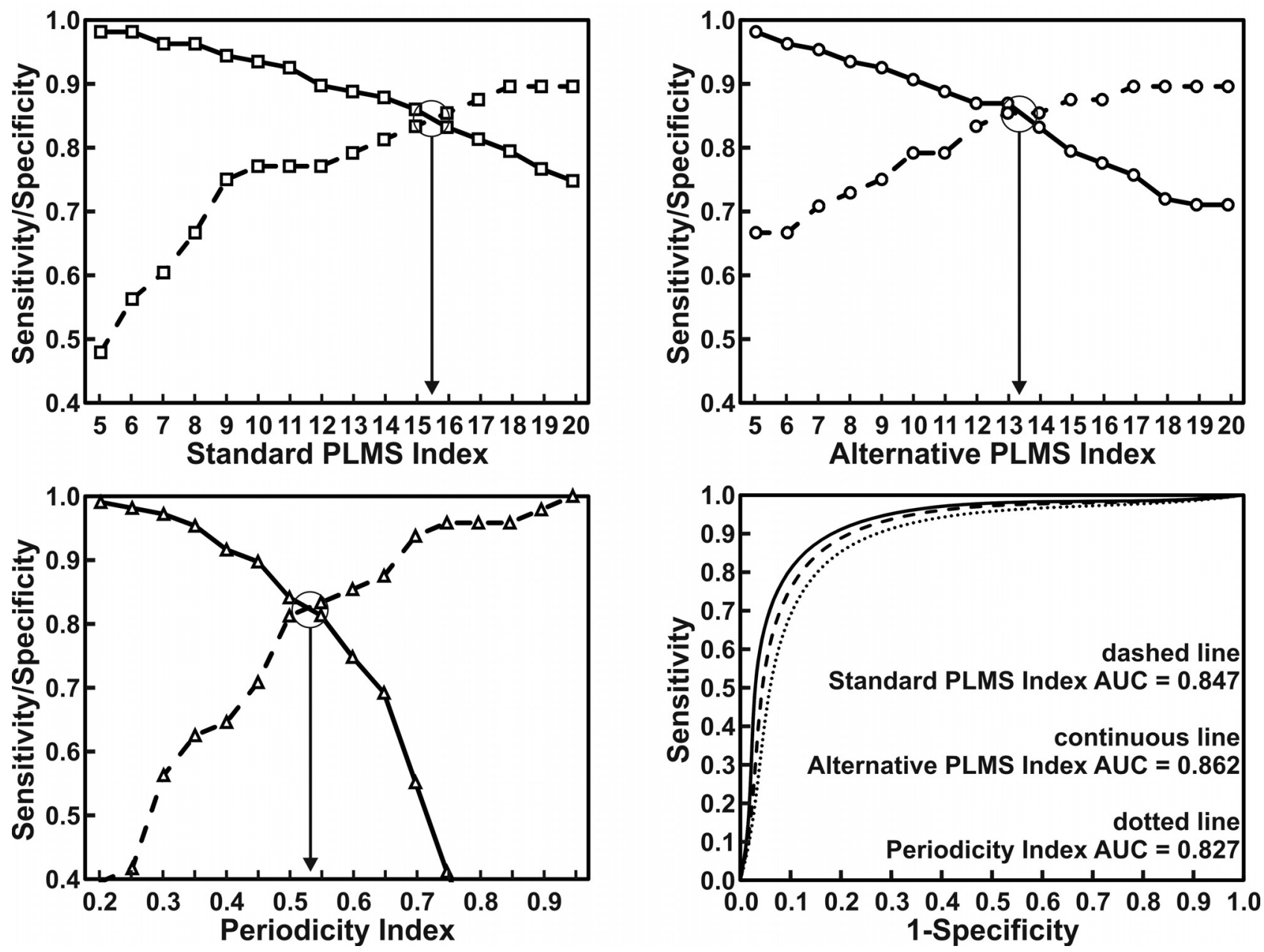